\author{
تأثير عنصر الكروم في نمو الطحلب الاخضر \\ Scenedesmus quadricauda (Turp.) de Breb. \\ عند زيادة تركيز النيتروجين \\ ألطاف عبل الواحد عيسى الراوي*

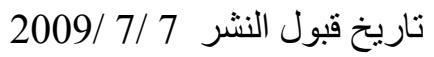

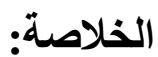

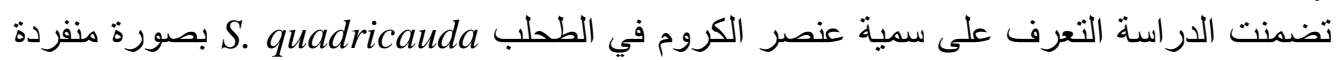

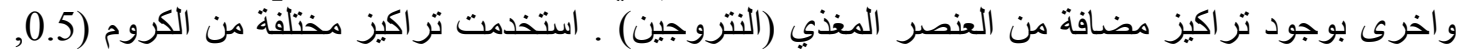

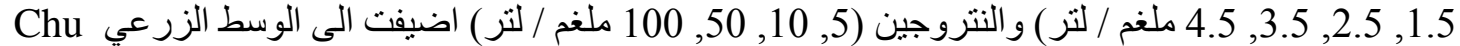

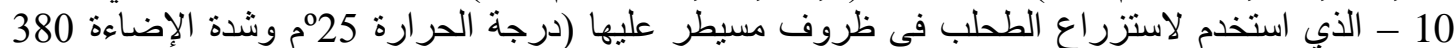

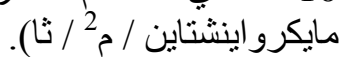

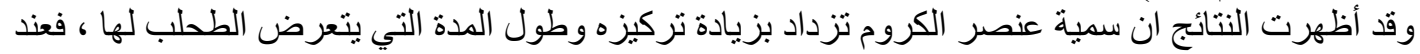

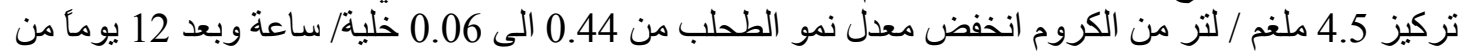

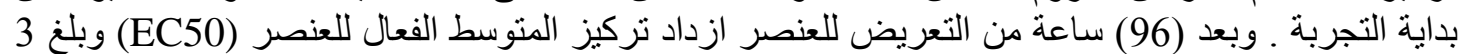

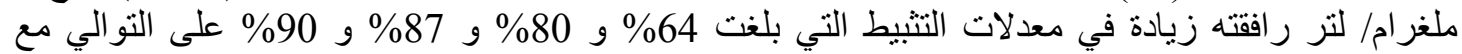

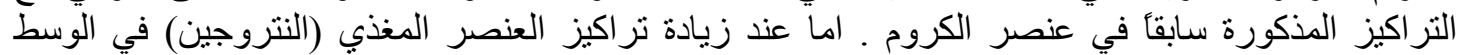

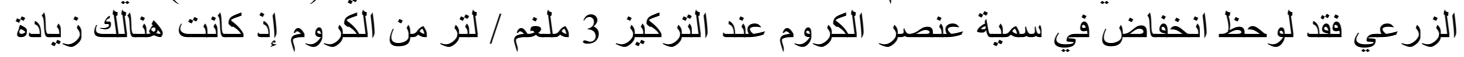

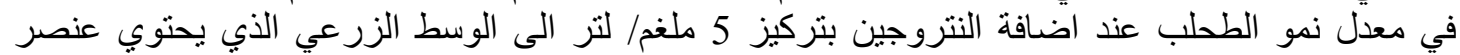

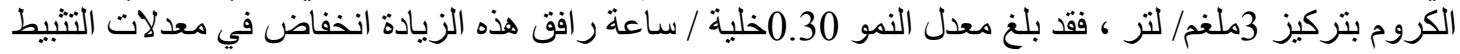

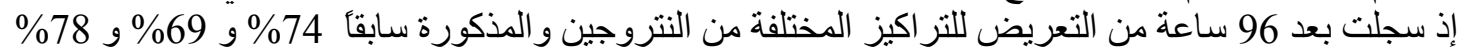
و 70\% و 69\% على التو الي وقد رافق هذا كله زيادة كثافة الخلايا وبشكل ملحوظ مما يعطي مؤشر آ على تقليل سمية عنصر الكروم بوجود النتروجين .
\end{abstract}

الكلمات المفتاحية: Green algae, Scenedesmus quadricauda, Heavy metal, Chromium

وطويلة المدى في الحياة المائية [5] ، وأن تلوث

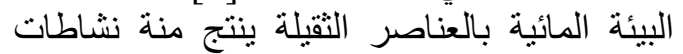

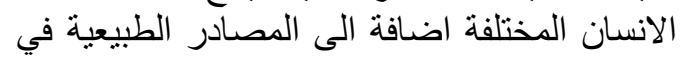

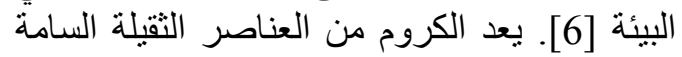
وهو فلز صلد ابيض اللون مزرق تبلغ كنافئنه 702

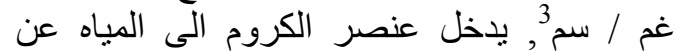
طريق تصريف مياه الفضلات الناتجة عن الطناء الطباء

الكهربائي وصناعة الجلود و إنتاج الصبغات النات [7].

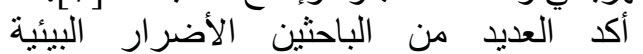

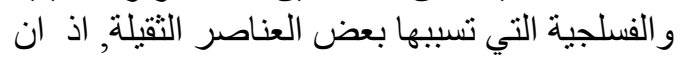

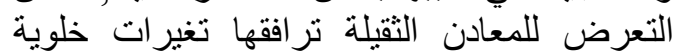

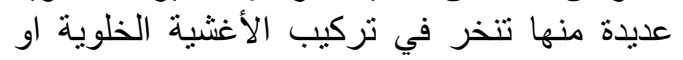

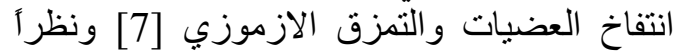
لأهمية الطحالب في البيئة المائية لإنها تمثل القاعية القاعدة

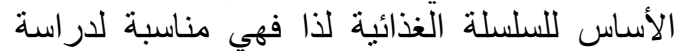

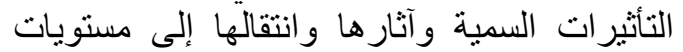

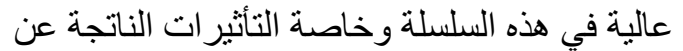

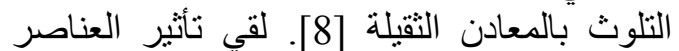
الثقبلة في الطحالب أهنمام الباحثين ومن أهم هذه

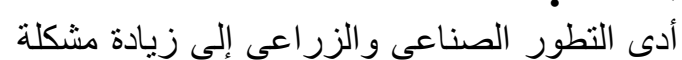
التلوث المائي الذي أدى الى تهديد الإلى الاحياء المائية ومنها الطحالب إذ تمثل الأخيرة المنتجات الألئ الأولية

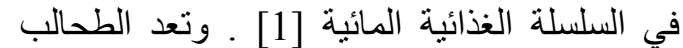

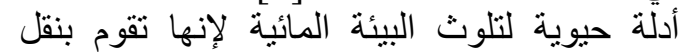
الملوثات الى الأحياء المائية في المستوية الأنيات الغذائية

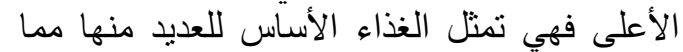

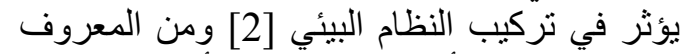

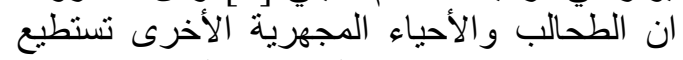

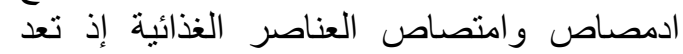
مرشحات حيوية لكثير من الملوثات الأخرى المئي

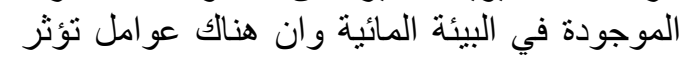

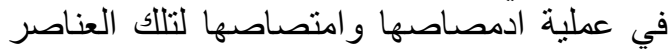
مثل الرقم الهيدروجيني وتركيز الكتلة الحية ونوع العنافئ العناصر الثقيلة والايونات المتنافسة وكذللك نوع ونرع

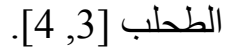

إن ملوثات البيئة المائية مثل العناصر الثقيلة

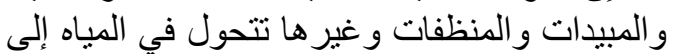
صور أكثر تعقيدأ مسببة نأثيرات سمية فية قصيرة

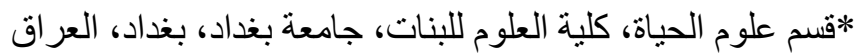


3.5 و 4.5 ملغ / لتر ، كما حضر محلول قياسي

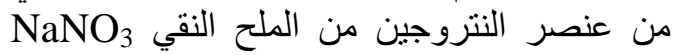
بتركيز 1000 ملغم / لتر ومنه حُضِّرَت التنر اكيز 5 و 10 و 50 و 10 ملغ / لترك و لتر.

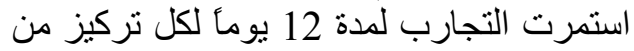

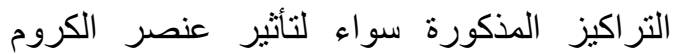

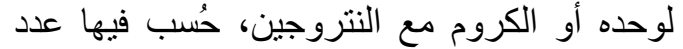
خلايا الطحلب من خلال حساب العدد الكلي للخايلايا

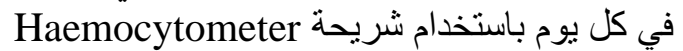
[17] وقيست الكثافة الضوئية أيضاً باستخدام جهاز

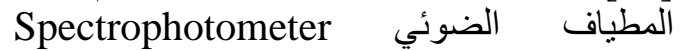
بتصفير الجهاز على طول موجي 540 نانوميتر ولمدة 12 يومأ أيضاً ، ومن حساب كثافة الطحلب لئب تم حساب معدل النمو Growth rate (M) وزمن التضاعف Doubling time (G) حُسب كذلك معدل التثيط Inhibition rate , بالاعتماد على Reynolds [18 [بحسب المعادلات الآتية:-

$$
\text { إذ ان:- }
$$

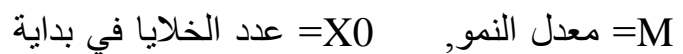
التجربة (خلية / مليلتر) ),

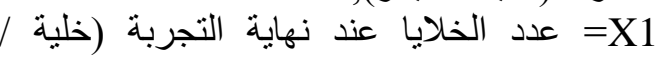
مليلتر), ال الزمن (يوم)

$$
\mathrm{G}=\frac{\ln \mathrm{n}_{2}}{\mathrm{M}}
$$

$0.693=\ln _{2}$, من التضاعف, = = معدل النمو=

وحسب معدل التثبيط بوصفه لنسبة مئوية [19] بتطبيق المعادلة

$$
\text { Inhibition \% }=1-\frac{\mathrm{XT}}{\mathrm{XE}} \quad \mathrm{X} 100
$$

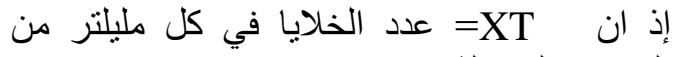
المز إن المعاملة

XE السيطرة

تم تحويل النسب المئوية للتثبيط ولكل معاملة الى وحدات احتمالية [20] لحساب التركيز Median Effective المتوسط الفعال EC50 Concentration تر اكيز عنصر الكروم إلى قيم لو غارتمية تم بعدها حساب التركيز المتوسط الفعال خلال 24, 72, 72, 96 ساعة لعنصر الكروم عند استخدامه لوحده من دون نتروجين، وفي تجارب إضافة النتروجين بالتر اكيز
الثأثيرات ثأثير العناصر في معدل التنفس [9

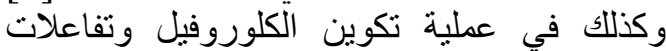
البناء الضوئي [10] فضلا عن نأثير ها في عدة

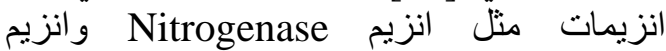
ونام Phosphatase

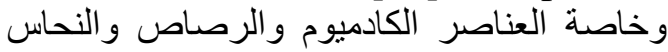
بصورة مجتمعة في نمو طحلب S.quadricauda بالاعتماد على الكتلة الحرية ممثلة بالعدد الكلى

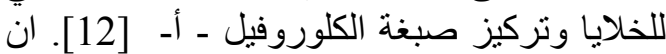
الهدف من الدراسة الحالية هو معرفة مدى سمية الكية

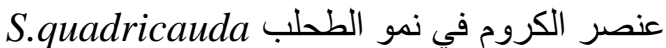
عندما يكون لوحده او عند اضافة تر اكيز مختلفة من الكن المغذيات النباتية مثل النتروجين الذي استخدم في هذه الدراسة. المواد وطرائق العمل:

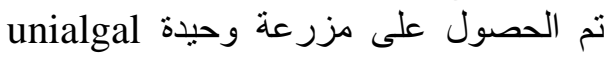

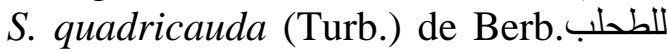
من عينة ماء من القناة الحولية لمجمع جامعة بخداد في الجادرية بطريقة التخفيف [13] [13, واتبعت طريقة باترسون [14] للحصول على عزلة نقية خالية من الأحياء المجهرية (14) (Axenic algal culture).

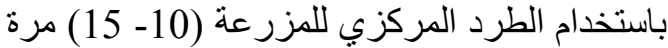
في ظروف معقمة ومن ثم اختيار العزلة بتنميتها عutrient agar على وسط المرق المغذي الصنا

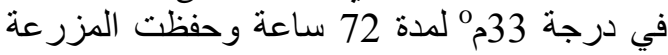
الخزينة Stock في ظروف فئ فئسية في المختبر.

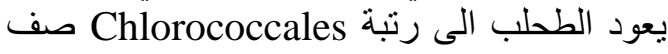
Chlorophyceae باحتو ائه على 2 - 8 من من الخلايا الكنطاولة التي

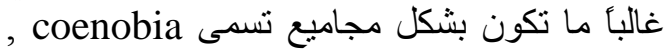

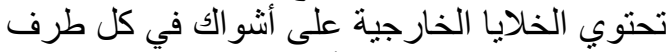
والداخلية لا تحتوي على أثنو الك ويصل قطر الخدر الخلية الواحدة 3 - 18 مايكرون وطولها 9 - 95 مايكرون [15]

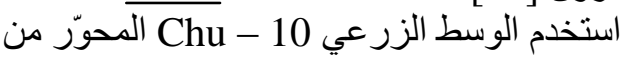
قاسم [16] وقد ضبط الأس الهيدروجيني بجهاز نوع DIG520 صنع شركة Wissenschftlich Tehnische-werkstath

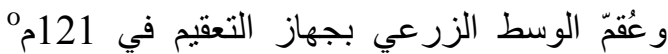
وضغط 1.5 جو لمدة 15 دقيقة وترك لييرد الليوم

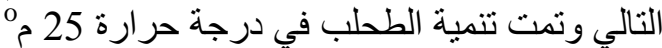

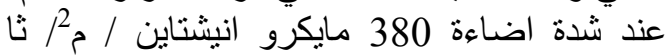

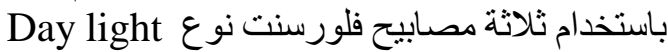

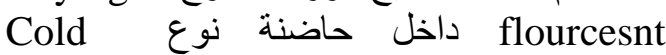
incubator BDH

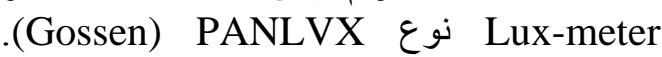
حضر محلول قياسي للعنصر الثقيل الكروم بتركيز 1000 ملغم / لتر من الكلح التركيز حُضرّت التراكيز 0.5 و 1.5 و و 2.5 و هن 
ومعاملة السيطرة الثانية التي تحتوي على 3 ملغم

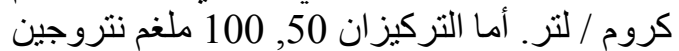

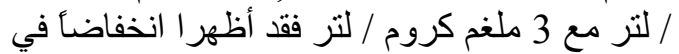

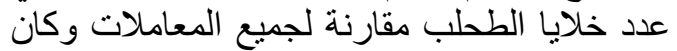
التأثثر أكثر وضوحأ منذ اليوم السابع الى غاية نهاية النية

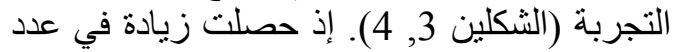

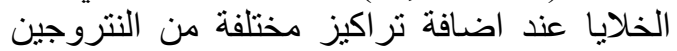
بوجود 3ملغم / لتر من الكروم مقارنة بالتركيز

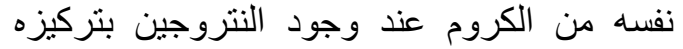
الاعتيادي في الوسط الزرعي الزئيط

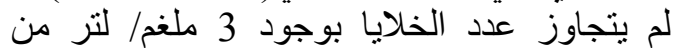

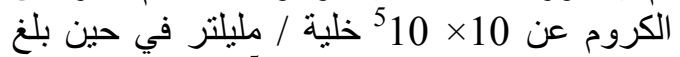

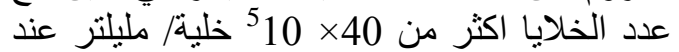

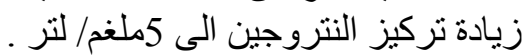

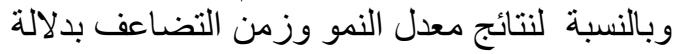

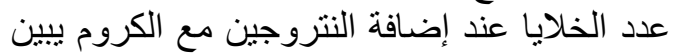
الجدول (3) زيادة في معدل النمو عند التركيزين

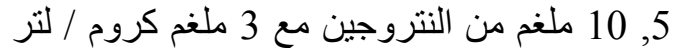

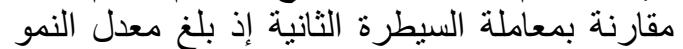

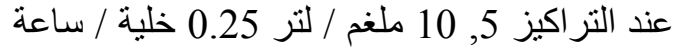
و 0.24 خلية / ساعة على التى التو الي مقارنة بالسبطرة

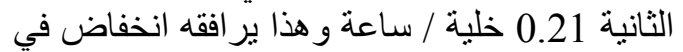

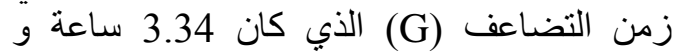

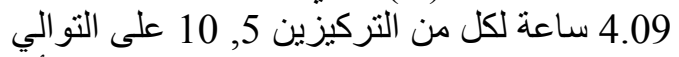

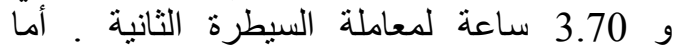

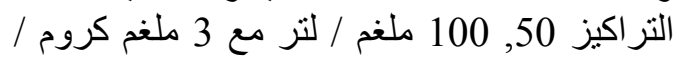

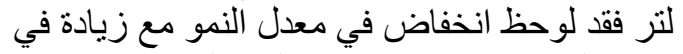

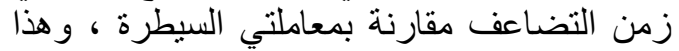

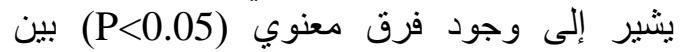

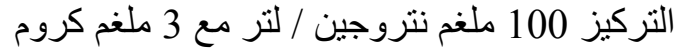

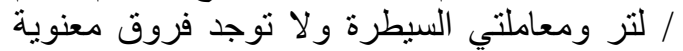

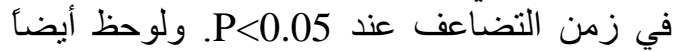

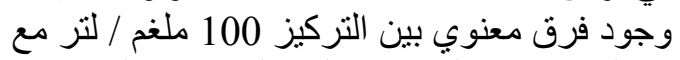
3 ملغم كروم / لتر ومعاملتي السيطرة و التركيزين ملترين

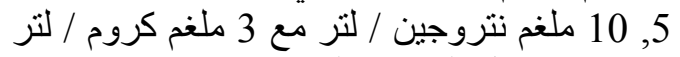

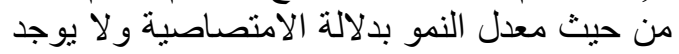

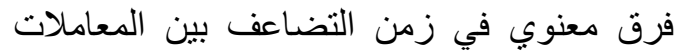

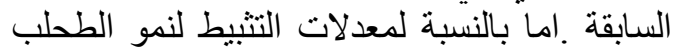

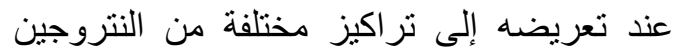

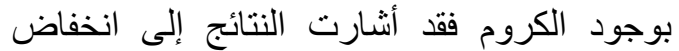
معدلات التنبيط خلال 96 ساعة لكل من التركيزين

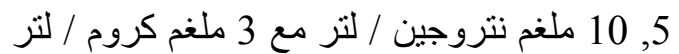

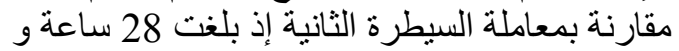

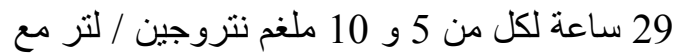

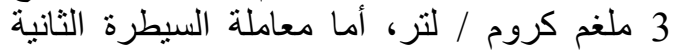

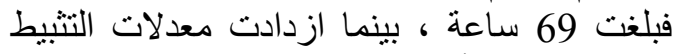
لبقية التر اكيز الأخرى من النتروجين (الجدول 4).
المذكورة سابقأ نم تحديد (EC50) لعنصر الكروم

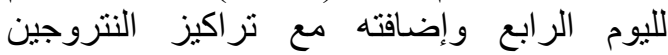
المستخدمة في الدراسة ـ تم تحليل النتائج إحصائياً باستخدام البرنامج الإحصائي 2004 SAS 2004 لتحليل

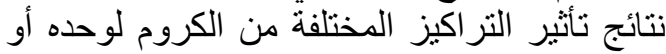

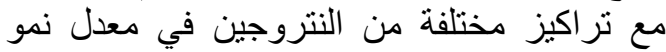
وزمن تضاعف خلايا الطحلب المدروس فئرئ [21] واستخدام اختبار الفرق المعنوي الأصغر عند تحقق مستوى الاحتمال (P>0.05).

\section{* تأثير عنصر الكروم في نمو الطحلب}

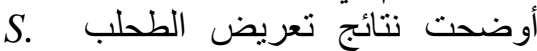
quadricauda

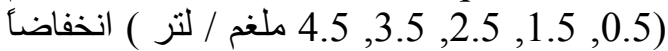

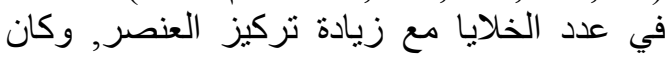
هناك فرق معنوي (P<0.05) بين هذه المعاملات

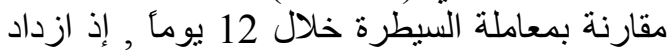

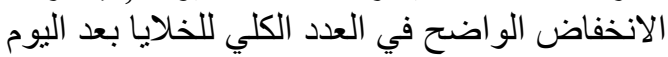

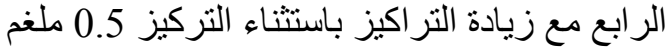

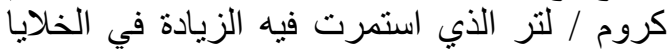

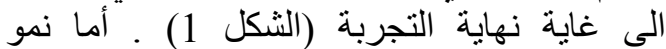
الطحلب بدلالة الامتصاصية فقد أظهرت النية النتائج نمطا مشابهاً تمامأ لعدد الخلايا و أظهر التركيز التران

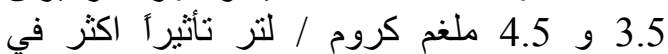
الطحلب من بقية التراكيز ابتداءً من اليوم الثناني اكتي و الثالث (الثكل 2).

انخفضت معدلات النمو بدلالة عدد الخلايا

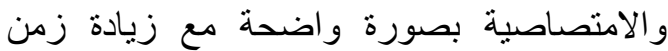

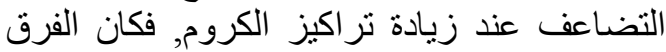

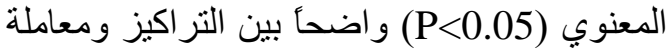

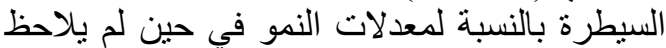
فرق معنوي بين معاملة السيطرة وبقية لئية التراكيز

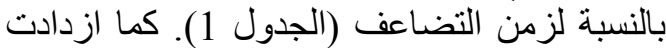

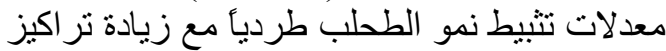

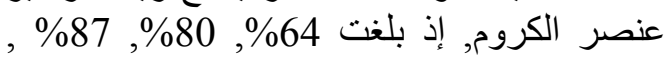
\%0 90 بعد 96 ساعة من تعريض الطحلب للتر اكيز

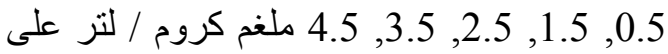

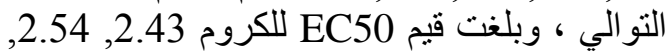

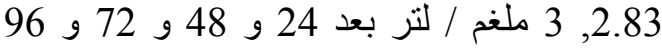

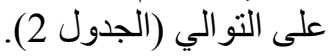

* ت تأثير زيادة تركيز النتروجين في سميّة الكروم أشنارت النتائج إلى زيادة في في أعداد خلايا

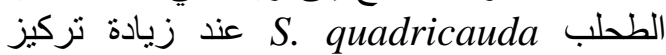

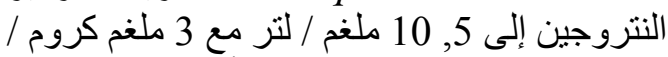
لتزر مقارنة بمعاملتي السيطرة الأولى التى التي تحنوي على الوسط الزرعي 10 - 10 Chu الاعتيادي الأولي 
شكل1: تأثير تر اكيز مختلفة من الكروم في نمو الطحلب Scenedesmus quadricauda المستزرع في الوسط الزروعي

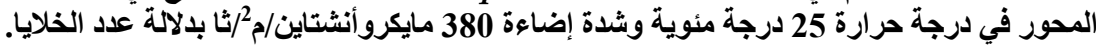

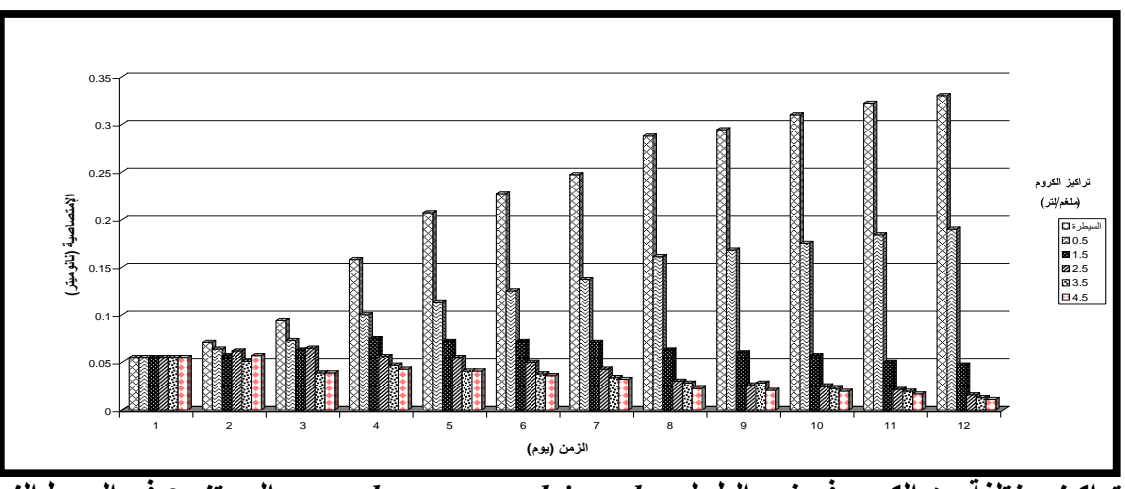

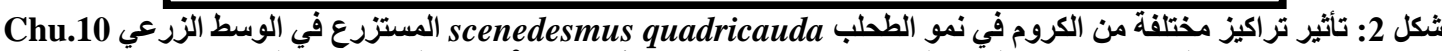
المحور في درجة حرارة 25 درجة مئوية وشدة إضاءة 380 مايكروأنشتاين/م²/ثا بدلالة الامتصاصية (540 نانوميتر).

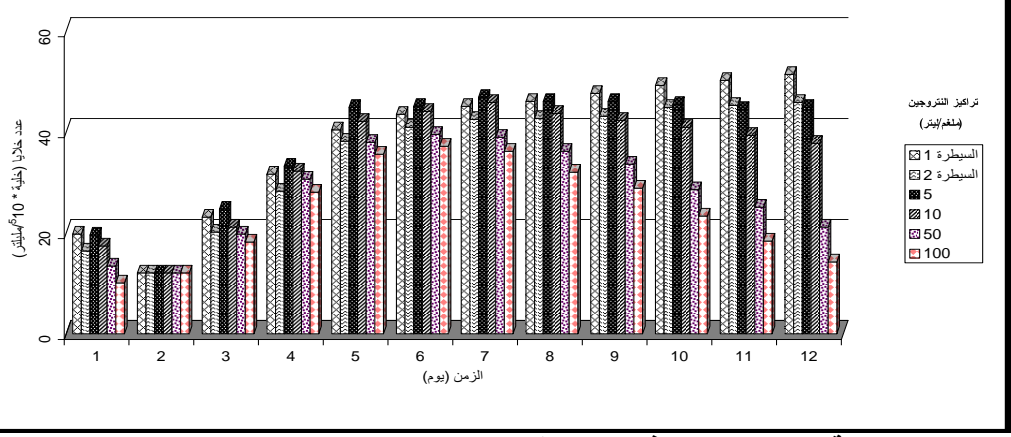

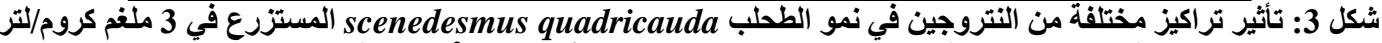

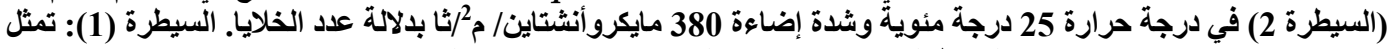

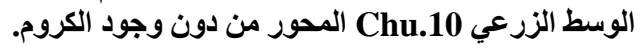

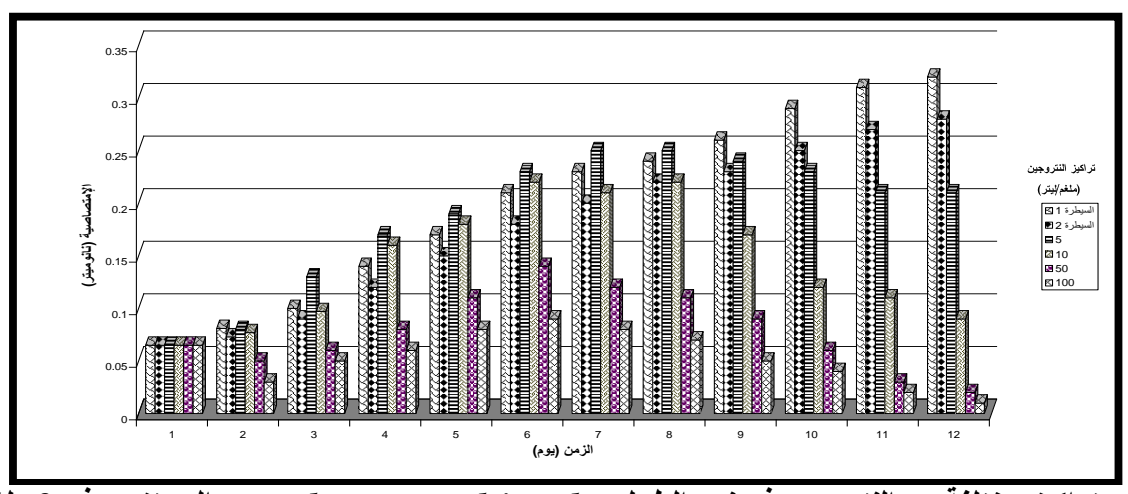

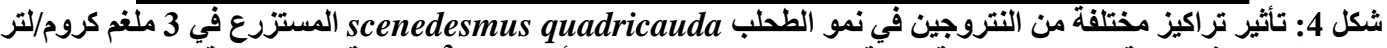

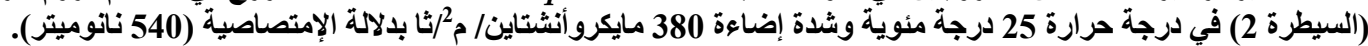

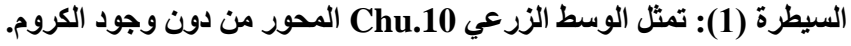




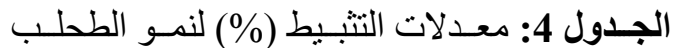
ع.quadricauda

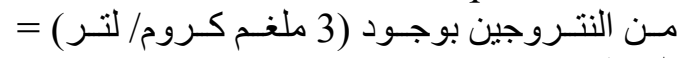

السيطرة (2) النيز

\begin{tabular}{|c|c|c|c|c|}
\hline ساعة & ساعة 72 & ساعة & ساعة & تر (ملغم/ لنتر) \\
\hline 25 & 30 & 35 & 40 & السيطرة)(1) \\
\hline 69 & 58 & 41 & 27 & السبطرة (2) \\
\hline 28 & 30 & 35 & 38 & 5 \\
\hline 29 & 31 & 30 & 31 & 10 \\
\hline 69 & 61 & 40 & 19 & 50 \\
\hline 67 & 58 & 34 & 11 & 100 \\
\hline
\end{tabular}

\section{* تأثير عنصر الكروم في نمو الطحلب}

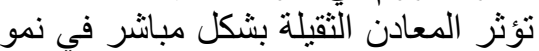

الطحلب من خلال تأثير ها في عملية تكاثر آلخلية

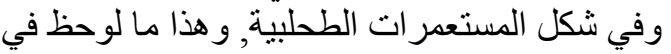

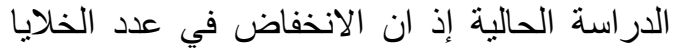

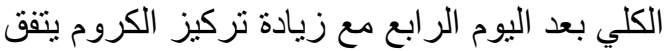

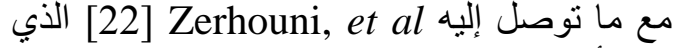

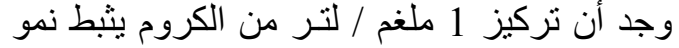
Chlorella و S. quadricauda الطحالب تركيز Chlamydomonas , pyrenoidosa reinhardii وان التر اكيز 1, 5, 10 ملغم كروم /

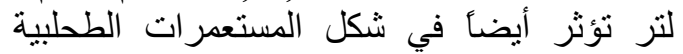

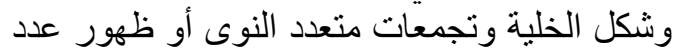

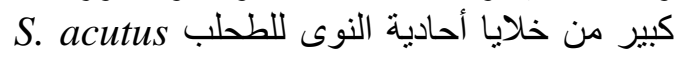

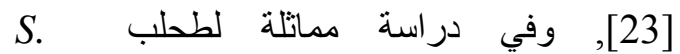
quadricauda

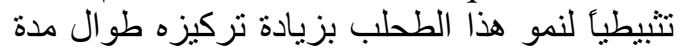
التعريض [24] ويعود السبب في التباين في ارتفاع

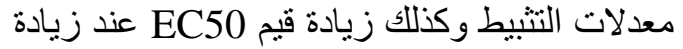

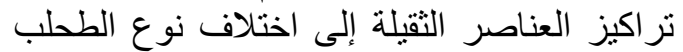

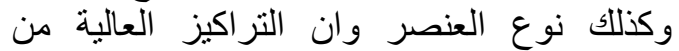
العناصر الثقيلة قد نؤثر في الفعاليات الايضية

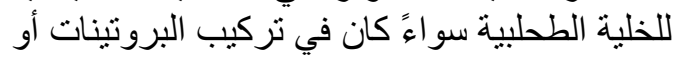

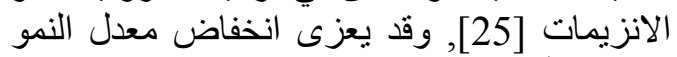

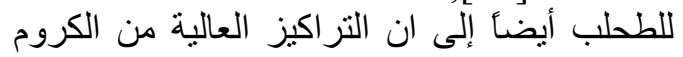
في الاوساط الصناعية قد تثبط عملية البناء الضوائي التئ التروم بنسبة 50\% [26]

* تأثير زيادة تراكيز النتروجين في سمية عنصر * الكروم

S. إن زيادة عدد خلايا طحلب

quadricauda عنصر نقيل الذي لوحظ في الدر اسة الحالية قد يعود

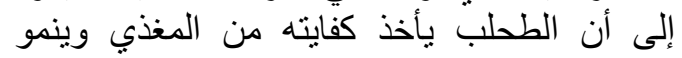

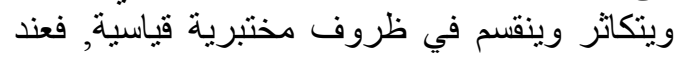
زيادة التراكيز إلى 5, 10 ملغم نتروجين / لتزئ

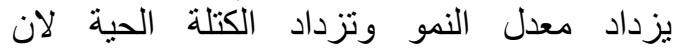
النتروجين يعد من المغذيات الأساسية لنمو لأنية
جدول1: معـدل النمـو وزمـن التضــاعف للطحلب Scenedesmus quadricauda

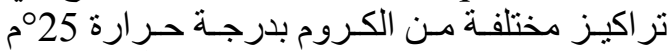

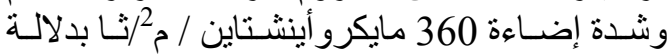
عدد الخلايا و الامتصاصية إهية

\begin{tabular}{|c|c|c|c|c|}
\hline \multicolumn{2}{|c|}{ بلالة الامتصاصبة } & \multicolumn{2}{|c|}{ بلالة عدد الخلايا } & التّراكيز \\
\hline زمن التضاعة) & (ظلية/ساعة) معلم & زمن التضاعف) & (خلية/ساعة) معلم & ملغ//تتر \\
\hline $\begin{array}{c}{ }^{\mathrm{ab}} 0.76 \pm \\
3.02\end{array}$ & ${ }^{\mathrm{a}} 0.06 \pm 0.24$ & ${ }^{\mathrm{a} a} 0.69 \pm 1.80$ & ${ }^{\mathrm{a} a} 0.19 \pm 0.44$ & السيطرة \\
\hline $\begin{array}{c}\text { ab } 0.83 \pm \\
4.89\end{array}$ & ${ }^{\mathrm{b}} 0.02 \pm 0.14$ & ${ }^{\mathrm{a} a} 0.72 \pm 2.52$ & ${ }^{\mathrm{b}} 0.12 \pm 0.30$ & 0.5 \\
\hline $\begin{array}{l}3.26 \pm \\
12.07\end{array}$ & ${ }^{\mathrm{c}} 0.05 \pm 0.05$ & $\begin{array}{c}{ }^{2} 20.10 \pm \\
3.98\end{array}$ & ${ }^{\mathrm{c}} 0.13 \pm 0.16$ & 1.5 \\
\hline $\begin{array}{c}\mathrm{b}_{21.12} \pm \\
9.85\end{array}$ & $* *$ & $\begin{array}{l}{ }^{\mathrm{a}} 2.32 \pm \\
11.21\end{array}$ & ${ }^{c} 0.10 \pm 0.11$ & 2.5 \\
\hline ** & $* *$ & $\begin{array}{l}{ }^{\mathrm{a}} 2.87 \pm \\
16.53\end{array}$ & ${ }^{\mathrm{c}} 0.12 \pm 0.10$ & 3.5 \\
\hline ** & $* *$ & $\begin{array}{l}\text { a } 2.10 \pm \\
31.86\end{array}$ & ${ }^{\mathrm{c}} 0.08 \pm 0.06$ & 4.5 \\
\hline 14.992 & 0.045 & ${ }^{\mathrm{ns}} 23.647$ & $* 0.117$ & LSD \\
\hline
\end{tabular}

الحروف الصغيرة المختلفة تدل على وجود فروق معنوية (P )

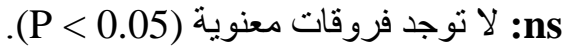
* * كانت الزيادة في عدد الخلايا في نهاية التجربة أقل من عددها عند بدأ التجربة.

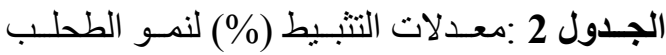
S.quadricauda من الكروم ولمدة 96 ساعة عنداع

\begin{tabular}{|c|c|c|c|c|}
\hline ساعة 96 & ساعة & ساعة & ساعة & تراكيز الكروم (ملغم/ \\
\hline 64 & 48 & 23 & 3 & 0.5 \\
\hline 80 & 50 & 26 & 28 & 1.5 \\
\hline 80 & 51 & 31 & 47 & 2.5 \\
\hline 87 & 64 & 26 & 49 & 3.5 \\
\hline 90 & 64 & 74 & 52 & 4.5 \\
\hline 3 & 2.83 & 2.54 & 2.43 & EC50 mg/l \\
\hline
\end{tabular}

جدول 3: معدل النمـو وزمسن التضـاعف للطحلب جالي

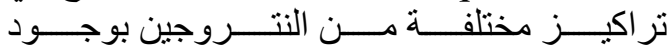

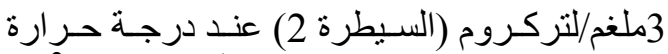

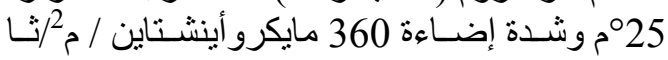

\begin{tabular}{|c|c|c|c|c|}
\hline \multicolumn{2}{|c|}{ بدلالة الامتصاصبية } & \multicolumn{2}{|c|}{ بلالة عدد الذلايا } & التُراكيز \\
\hline زمن التضاعفة & (خلية/ساعة نموة) & زمن التضاعة) & (خلية/ساعة) & ملغ//تتز \\
\hline $\begin{array}{l}0.74 \pm \\
3.65\end{array}$ & ${ }^{\mathrm{a}} 0.03 \pm 0.19$ & ${ }^{\mathrm{a}} 1.29 \pm 3.33$ & ${ }^{\mathrm{a}} 0.10 \pm 0.24$ & $\begin{array}{l}\text { السبطرة } \\
\text { (1) }\end{array}$ \\
\hline $\begin{array}{l}0.90 \pm \\
4.43\end{array}$ & $\mathrm{a} 0.03 \pm 0.16$ & ${ }^{\mathrm{a}} 1.26 \pm 3.70$ & ${ }^{\mathrm{a}} 0.06 \pm 0.21$ & $\begin{array}{l}\text { السبطرة } \\
\text { (2) }\end{array}$ \\
\hline $\begin{array}{l}{ }^{\mathrm{b}} 1.62 \pm \\
3.85\end{array}$ & ${ }^{\mathrm{a}} 0.07 \pm 0.21$ & ${ }^{\mathrm{a}} 1.56 \pm 3.34$ & ${ }^{\mathrm{ab}} 0.22 \pm 0.25$ & 5 \\
\hline $\begin{array}{l}{ }^{b} 9.57 \pm \\
4.43 \\
\end{array}$ & ${ }^{\mathrm{ab}} 0.08 \pm 0.16$ & $\mathrm{a}_{2.08} \pm 4.09$ & ${ }^{\mathrm{a}} 0.11 \pm 0.24$ & 10 \\
\hline $\begin{array}{l}{ }^{b} 9.57 \pm \\
8.11\end{array}$ & ${ }^{\mathrm{bc}} 0.20 \pm 0.06$ & ${ }^{a} 3.81 \pm 5.18$ & ${ }^{\mathrm{ab}} 0.10 \pm 0.18$ & 50 \\
\hline $\begin{array}{l}{ }^{b} 27.51 \pm \\
13.15\end{array}$ & ${ }^{\mathrm{c}} 0.27 \pm 0.05$ & $\begin{array}{c}{ }^{\mathrm{a}} 1.98 \pm \\
10.01\end{array}$ & ${ }^{\mathrm{b}} 0.13 \pm 0.12$ & 100 \\
\hline${ }^{\mathrm{ns}} 10.483$ & $* 0.126$ & Ns7.169 & $* 0.113$ & LSD \\
\hline
\end{tabular}

الحروف الصغيرة المختلفة تدل على وجود فروق

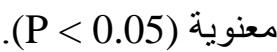
ns 
of Crop Culture, $16^{\text {th }}$ Urniv. Tech. Sydney. Pp.1-29.

4- Rohlaxck, T.; Pittman, E.; Borner, T. and Christoffer, K.2001. Effects of Cell- bound microcystins on survival and feedung of Daphnia spp. Env. Microbiol ., 67(8): 35233529.

5- Chatak, D.B and Hanar, S.K. 1993. Chronic sublethal effects of mixture of heavy metals cadmium , pesticide, DDVP, detergent parnol and Petroleum product N- lteptan, on fish. Env. and Ecol. 11(4): 778783.

6- Behra, R.; Landwehrjohann, R.; Vogel, K. ; Wagner, B. ans Sigg, L.2002. Copper and Zinc content of Periphyton from two rivers as afunction of dissolved metal concentration. Aquat. Sci.964: 300306.

7- Lamas, E.M.; Doares, M.J. and Oleveira, M.M. 1996. Effects of cadmium on growth of Euglena gracilis membrane lipids. Bra. Z.J. and Med. Biol., 29(8): 941- 949.

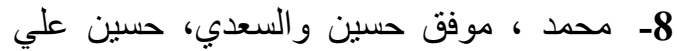

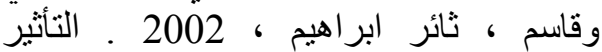
التراكمي لبعض العناصر الثقيلة في طحلب التبل

Scenedesmus quadricauda العر اقية لعلم الاحياء 2(1): 24- 31.

9- Hart, B.A. and Seaife, B. D. 1977. Toxicity and bioaccumulation of cadmium in chorella pyrenoidosa, Env. Res. 14: 401- 413.

10- Twiss, M. R. and Nalew Asko, C. 1992. Infliunce of phosphorous nutrition on copper toxicity to three strains of Scenedesmus acutus (Chlorophyceae).J. Phycol. 98: 291- 298.

11-Bettger, W.J. and O'dell, B.L. 1981. A critical physilogical role of zinc in the structure and function of bio membrance life. Sci, 28:142143.

12- السعدي، حسين علي وقاسم، ثائر ابراهيم

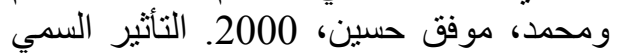

لخليط من بعض المعادن الثقيلة في الطحلب
الطحالب وان احتياجات الطحالب لها تختلف

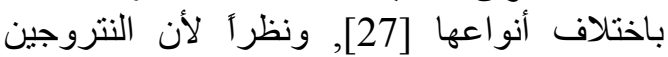

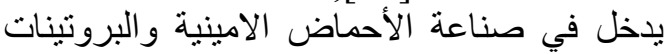

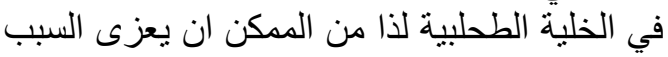

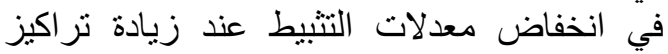
النتروجين بوجود الكروم إلى ان التيط التراكيز العالية

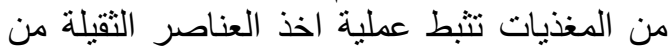

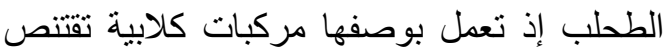

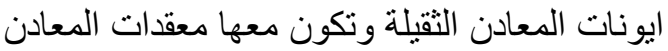
ومن ثم تقلل من سمية هذه المعادن عند و جودها فيا في

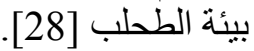

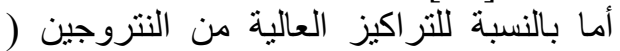

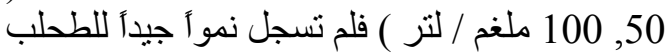

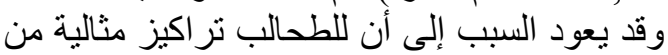

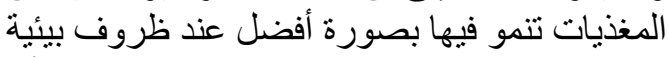

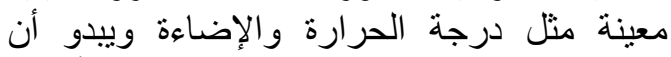

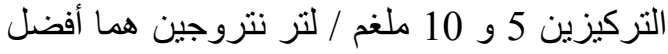

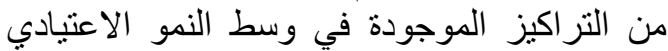

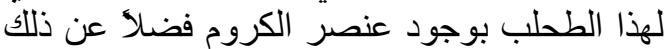

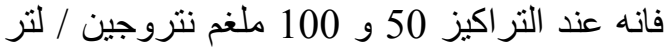

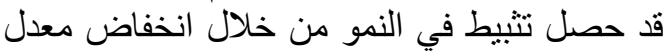
انقسام الخلية وقد يشير هذا إلى أن زيادة نركيز

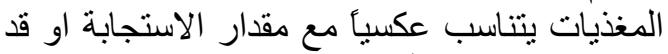

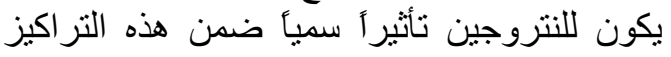

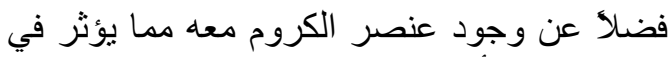

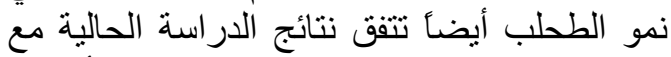

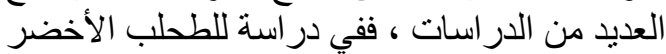

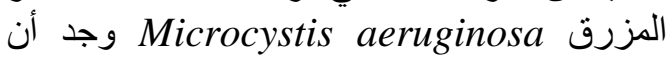
هنالك زيادة في معدلات نمو الطحلب عند إضافة

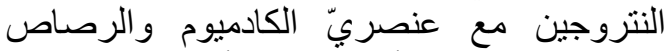
[29], وفي دراسة أخرى تؤكد أن المغذيات مثل الترئ النتروجين تقلل من سمية العناصر الثقيلة أما بشكل

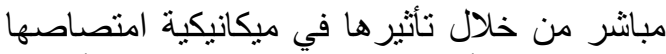

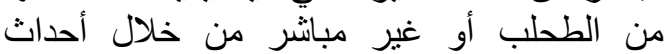
تغير ات فسيولوجية للخلية الطحلبية [30].

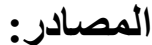

1- Fies, S.C.; Fleming, K.; Korbhals, E.; Searte, G.; Reynolds, L. and Karner, D. 1999. Modification to the algal growth inhibition test for use a regulatory assay. Philadelphia, PA, USA.

2- Gunatilake, A. and Diehl, P. 2000. A brief of chemical and biological continuous monitoring of rivers in Europe and Asia- Plenum press, N.Y.

3- Matin, Aiw. 2002. CarbonFertilisers Link. Research Station 
cadmium uptake by three green algae species isolated from urban westwater. Ecotoxicol. Env. 19(2): 255- 261.

23- Al. Saadi, H.A. and Kassim, T.I. 2002. Use of algae scenedesmus acutus in control and treatment of some heavy metals. Proc. Int. Symp. Env. Poll. Control and Waste Manag., : 147- 154.

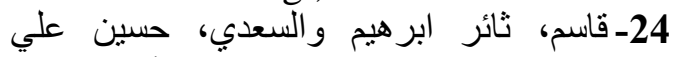

ومحمد، موفق حسين، 2000 سُمية بعض المين

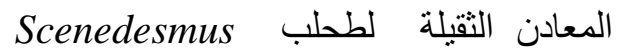

quadricauda

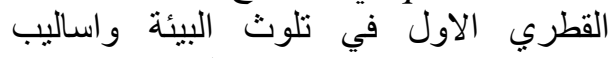

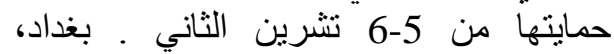
العراق، 439- 452.

25-Sunda, W.G. 1990. Trace metal interactions with marine phytoplankton. Biol. Oceano. 6:411- 442.

26-Eisler, R. 2000. chemical Risk Assessment. Vol., 1. Lewis Publ. U.S.A. pp.99-311.

27- Allen, H.E. and Kurmer, J.R. 1972. Nutrient in nature water. Jhon Wiley and Sons, Inc, Tornto.

28-Florence, A.M. 2007. Heavy metals. Contamination and toxicity. Stockholm University Sweden.

29-قاسم ، ثائر ابراهيم وحسن، فكرت مجيد

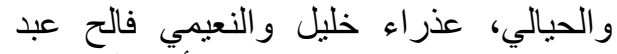

حسن وأخلاص، حسن،2004. تأثئير الكادميوم

والرصاص في نمو طحلب Microcycstis

aeruginosoa

المؤتمر الدولي الثاني للتنمية والبيئة في الوطن التون

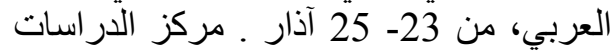

والبحوث البيئة ، جامعة أسيوط، مصرك، 23

.29-

30-Bajguza, A. 2000. Blockage of heavy metals accumulation in Chlorella vuylgaris. Celby 24epbrassinolidionplant. Physiology. Bioch., 38: 797- 801.
Scenedesmus quadricauda

ابحاث البيئة والتنمية المستدامة 3(2): 39-

.46

13-Belcher, H. and Swale, E. 1982. Culturing algae in statute of terrestrial(Ecology). Cambrige England.

14- Patterson, G. 1983. Effect of heavy metals on fresh water chlorophyta, Ph.D. Thesis, Univ .Dur ham, pp.212.

15- Kassim, T.I. ; Al-Saadi, H.A. and Salman, N.A. 1999. Production of some Phytoplankton and zooplankton and their use as live food for fish larva. Iraqi J. Agric. (Special issue), 4(5):188-201.

16- Kassim, T.I.; Al- Saadi. H.A.; Salman, N.A. and Dally, F.A.A. 2002. Influnce of temperature, Light intensity and nutrients concentration on the growth of of Scenedesmus acutus Meyen. Iraqi. J.Biol. 28: 291- 296.

17- Martinez, M.R.; Chakroff, R.P. and Pantastico, J.B. 1975. Note on direct Phytoplankton counting technique using the Heamocytometer. Phil. Agric, 57: $1-12$.

18-Reynolds, C.S. 1984. The ecology of freshwater Phytoplankton. Cambridge Univ. Press. pp.384.

19- Nyholm, N.1985. Response Variable in algae growth inhibition test biomass or growth rate. Wat. Res., 19(3): 273- 279.

20-Matsumura, F.1975.Toxicology of insecticides. Plenum press. New York.

21-SAS. 2004. SAS user's guid for personal computers. Release 7.0. SAS Inst. W.C.USA.

22-Zerhouni, R.A; Bouya, D; Ronneau, G. and Cara, J. 2004. Phosphate, nitrogen, chromium and 


\title{
Effect of Chromium on Growth of green ALGA SCENEDESMUS QUADR ICAUDA (TURP.) DE BERB. With increase of nitrogen concentration
}

\begin{abstract}
Altaf Abid alwhed Al-Rawi*
*Department of Biology, college of science for women, university of Baghdad , Baghdad Iraq.

Abstract:

The study was conducted to identify the toxicity of chromium on scenedesmus quadricauda algae alone and in the presence of nutrient metal (Nitrogen). Different concentrations of chromium $(0.5,1.5,2.5,3.5,4.5 \mathrm{mg} / \mathrm{L})$ were used and the presence nitrogen is $(5,10,50,100 \mathrm{mg} / \mathrm{L})$ on cultur media(chu-10) with used for cultivation of the algae in controlled conditions $\left(25^{\circ} \mathrm{C}\right.$, light intensity $380-\mu \mathrm{E} / \mathrm{m}^{2} / \mathrm{s}$. The results showed increasing in the toxicity of the metal when is alone, excess of concentrations an time of exposure. The growth rate decreased from 0.44 to 0.06 cell/ hour after 12 day of the biging of the experiment and of concentrate $4.5 \mathrm{mg} / \mathrm{L}$ of chromium.

The intermediate active concentrations of the chromium(EC50) was increased after 96 hours from the exposure, which it reached alevel at $3 \mathrm{mg} / \mathrm{L}$, which the increase of rate of inhibition which became 64, 80, 87 and $90 \%$ respectively with above mentioned concentrations of chromium. While after increasing the nitrogen concentrations in the culture media, an decrease was detected in the toxicity of the chromium at $3 \mathrm{mg} / \mathrm{L}$. Level through the increase in growth rate reached 0.30 cell /hour after the addition of $5 \mathrm{mg} / \mathrm{L}$ of Nitrogen to the culture media which contain $3 \mathrm{mg} / \mathrm{L}$ of chromium. This increase was also a companied by a decrease in rate of inhibition at a level of $74 \%, 69 \%, 78 \%, 70 \%$ respectively with the above mentioned treatment concentrations. From the above results it could be concluded that the toxicity of chromium may be declined under the effect of the Nitrogen.
\end{abstract}

